\title{
Determination of Volatile Compounds of Illicium verum Hook. f. Using Simultaneous Distillation-Extraction and Solid Phase Microextraction Coupled with Gas Chromatography-Mass Spectrometry
}

\author{
Wenbo Zhang ${ }^{1}$, Yan Zhang ${ }^{2 *}$, Xueyan Yuan ${ }^{1}$ and Erlin Sun ${ }^{3}$ \\ ${ }^{1}$ School of Life Sciences and Technology, Xinxiang Medical University, Xinxiang, Henan 453003, ${ }^{2}$ Key Laboratory of Food \\ Nutrition and Safety (Tianjin University of Science and Technology), Ministry of Education, Tianjin 300457, ${ }^{3}$ Tianjin Institute of \\ Urology, Department of Urology, The Second Hospital of Tianjin Medical University, Tianjin 300211, PR China \\ *For correspondence: Email: cpzyyan@126.com; Tel: +86-13752353154
}

\begin{abstract}
Purpose: To determine the characteristic aromatic constituents of star anise (Illicium verum Hook. f.) and identify its main aroma compounds for sensory evaluation.

Methods: The volatile compounds of star anise were prepared by simultaneous distillation-extraction $(S D E)$ and the compounds were identified by gas chromatography-mass spectroscopy (GC-MS). The flavor compounds were confirmed by gas chromatography-olfactometry (GC-O). Their flavor dilution factor (FD) values were determined using aroma extract dilution analysis (AEDA). Finally, standard samples that meet the requirements for sensory evaluation were extracted by the solid-phase microextraction (SPME) to further validate characteristic aroma molecules.

Results: Forty seven compounds were confirmed, with trans-anethole, estragole, and anisaldehyde the main aroma components. The content of trans-anethole was the highest (75.76\%), followed by linalool (1.44\%). Limonene (1.01\%) and 4'-methoxypropiophenone (0.72\%) were the lowest.

Conclusion: Combining SDE coupled with AEDA and SPME coupled with GC-MS is beneficial for identifying characteristic aroma compounds for star anise, such as trans-anethole, linalool and limonene in terms of FD value. The findings may be useful for star anise quality control in the food industry.
\end{abstract}

Keywords: Aroma, Flavor dilution factor (FD value), Gas chromatography-olfactometry, Simultaneous distillation-extraction, Sensory evaluation, Star anise, Illicium verum, Trans-anethole, Linaloo, Limonene

\begin{abstract}
Tropical Journal of Pharmaceutical Research is indexed by Science Citation Index (SciSearch), Scopus, International Pharmaceutical Abstract, Chemical Abstracts, Embase, Index Copernicus, EBSCO, African Index Medicus, JournalSeek, Journal Citation Reports/Science Edition, Directory of Open Access Journals (DOAJ), African Journal Online, Bioline International, Open-J-Gate and Pharmacy Abstracts
\end{abstract}

\section{INTRODUCTION}

Flavor substances are volatile aromatic compounds from food and spices that emit aroma during cooking and processing [1]. Star anise, a characteristic Chinese spice from the fruits of Illicium verum Hook. f. (Illiciaceae), contains 5 - $8 \%$ volatile essential oil, and is widely used in household, food industry and pharmaceutical industry $[2,3]$. I. verum produces star-shaped and reddish-brown fruits, consisting of six to eight follicles arranged in a whorl [2]. To investigate its volatile constituents and assess their contributions to flavor, it is necessary to extract and analyze the compounds. In this study, we applied two methods to collect volatile compounds, i.e. SDE and solid-phase microextraction (SPME). Combining these two methods can enhance repeatability and 
representativeness for analyzing typical spice volatile molecules.

\section{EXPERIMENTAL}

\section{Materials and chemicals}

Star anise was purchased from a local supermarket and identified by Professor Ping $\mathrm{Xu}$ of Xinxiang Medical University. A voucher specimen (No. ZWB-1) was deposited in the central laboratory of Xinxiang Medical University. The SPME manual fiber assembly and SPME fibers (carboxen/polydimethylsiloxane, $75 \mu \mathrm{m}$; and divinylbenzene/carboxen/polydimethylsiloxane, $50 \mu \mathrm{m}$ ) were purchased from Supelco Inc (Bellefonte, PA, USA). Other chemicals used were of analytical grade.

\section{Aroma components preparation by SDE}

Pulverized star anise ( $20 \mathrm{~g}, 75 \mu \mathrm{m}$ particle size) and a small amount of zeolite were mixed with distilled water $(500 \mathrm{~mL})$ in a round-bottomed flask $(1000 \mathrm{~mL})$. The temperature of the circulating water system was operated at $8{ }^{\circ} \mathrm{C}$ and the temperature of heating was controlled with an electric heating mantle. Then, the flask with sample mixture was jointed with a Simultaneous Distillation Extractor (Anhui Tianchang Youxin Electrical Equipment Co Ltd, China) and extracted with ethyl ether $(40 \mathrm{~mL})$ in an extraction bottle of the SDE apparatus at 40 ${ }^{\circ} \mathrm{C}$ for $3 \mathrm{~h}$. The extract was concentrated by nitrogen purging in $40{ }^{\circ} \mathrm{C}$ water bath to remove ethyl ether and dried by sodium sulfate for the subsequent gas chromatography-mass spectroscopy (GC-MS) and gas chromatographyolfactometry (GC-O) analysis.

\section{GC-MS and GC-O analyses}

GC-MS analysis was performed on an Agilent 6890N GC-5973iMS GC-MS system (HP Inc, USA) equipped with a flame ionization detector and a fused silica capillary column HP-5MS (5\% phenyl-methylpolysiloxane, $30 \mathrm{~m} \times 0.25 \mathrm{~mm}$ ID, $0.25 \mu \mathrm{m}$ film thickness). Helium was used as carrier gas at a flow rate of $1 \mathrm{~mL} \mathrm{~min}{ }^{-1}$. Injection port temperature was $250{ }^{\circ} \mathrm{C}$. Column temperature program was as follow: $50{ }^{\circ} \mathrm{C}(2$ min) isotherm, increased to $280{ }^{\circ} \mathrm{C}$ at a rate of $10^{\circ} \mathrm{C} \mathrm{min}{ }^{-1}$ and held at $280{ }^{\circ} \mathrm{C}$ for $30 \mathrm{~min}$. The mass spectrometer was operated in the electron impact ionization mode (+El, $70 \mathrm{eV})$. The compounds collected by SDE were identified by searching NIST02.1 database of the MSDChem workstation. GC-O analysis was performed on an Agilent 7980 gas chromatograph interfaced to an Alphamos sniffer device (Alpha-Mos Corporation,
France). The split ratio of the effluent into the Alpha-Mos sniffer was 10:1. Mass spectra were recorded in the range of $\mathrm{m} / \mathrm{z} 30-500 \mathrm{amu}$ in the full scan acquisition mode. The compounds were identified by GC-MS by comparing with retention index (RI) and aromaticity of standard compounds.

\section{AEDA method}

Samples prepared by SDE after removal of ethyl ether and water were serially diluted with ethyl ether at a ratio of 1:3, 1:9, 1:81 and 1:243. Diluted samples $(2 \mu \mathrm{L})$ were sequentially injected for GC-O analysis. No further dilution was performed, until the human assessors of our group at the sniffing port of GC-O could not detect the odor. FD factor was defined as the largest dilution ratio for each aroma component. AEDA was operated by three assessors recording odor character and time from the sniffing port of GC-O instrument, which should be consistently validated by two assessors [4].

\section{Sensory assessment of star anise}

Several characteristic aromas were suggested to be chosen to assess star anise after carefully evaluating standard star anise which was confirmed by Professor $\mathrm{Xu}$ and a panel of inspectors in our group by visual and chemical identification according to Chinese Pharmacopoeia (Bajiaohuixiang, Fructus Anisi Stellati, 2005 edition). Sweet aroma (30\%), alcoholic aroma $(20 \%)$, fruity aroma $(20 \%)$, woody aroma $(20 \%)$ and caramelic aroma (10 $\%$ were tentatively chosen as standards for grading and evaluating star anise. Total score of sample achieving 80 or more was qualified. According to the quantitative description analysis (QDA) sensory assessment rules, five or more inspectors were involved in the evaluation [5].

\section{Extraction using SPME fibers and GC analysis}

The star anise sample was pulverized with a pestle and mortar, then sieved through a $180 \mu \mathrm{m}$ mesh to obtain powder $(1 \mathrm{~g})$ sealed in a sample vial $(10 \mathrm{~mL})$. Load the SPME fiber assembly by exposing it to the sample inside the sample vial for $30 \mathrm{~min}$ to absorb at $25^{\circ} \mathrm{C}$. Then, secures the SPME fiber assembly in the injection port during the thermal desorption process with care. The gas chromatograph was equipped with an HP$5 \mathrm{MS}$ capillary chromatography column $(30 \mathrm{~m} \times$ $0.25 \mathrm{~mm}$ ID, $0.25 \mu \mathrm{m}$ film thicknesses, J \&W Scientific, Folsom, CA, USA). Column temperature program was set as follow: $50^{\circ} \mathrm{C}$ for 2 min isotherm, increased to $280^{\circ} \mathrm{C}$ at a rate of 
$10^{\circ} \mathrm{C}$ per min and held for 30 min. Injector port temperature was $250{ }^{\circ} \mathrm{C}$ and the split ratio was 10:1. Carrier gas was helium at a flow rate of 1 $\mathrm{mL} \mathrm{min}^{-1}$. The temperatures of electron impact (EI) ion source, quadrupole and transfer line were set at 230,150 and $280^{\circ} \mathrm{C}$, respectively. Ionization energy was set at $70 \mathrm{eV}$.

\section{RESULTS}

\section{Star anise aroma compounds}

The yield of volatile components of star anise obtained by SDE was approximately $2-3 \%$. Seventy-eight compounds were analyzed from SDE extracts and the total ion current chromatogram acquired (Figure 1). Forty-seven compounds were confirmed by searching NIST 02.1 mass spectral library in MSD ChemStation. Relative content of each compound in essential oil was calculated by peak area normalization method. These compounds accounted for more than $90 \%$ in total star anise aroma molecules, including trans-anethole (75.76 \%), panisaldehyde (8.65 \%), estragole (4.70\%), farnesol (3.26\%), limonene (1.01\%), linalool $(1.44 \%)$, caryophyllene (1.03 \%) and 4'methoxypropiophenone $(0.72 \%)$.

\section{Characteristic aroma molecules of star anise}

GC-MS analysis results indicated that transanethole, estragole, $p$-anisaldehyde and farnesol account for more than $90 \%$ in total star anise volatile components obtained with SDE. These compounds were mostly composed of a phenyl parent ring and alkene bond, with similar physiochemical properties, such as density (approx. 0.96 - 1.1), boiling point (approx. 210 $250{ }^{\circ} \mathrm{C}$ except for farnesol, $310^{\circ} \mathrm{C}$ ) and aroma. Overall, characteristic star anise aroma was produced by a group of compounds with similar star anise-like aroma, such as trans-anethole, $p$ anisaldehyde, farnesol and estragole (Figure 2).<smiles>C/C=C/c1ccc(OC)cc1</smiles>

trans-anethole<smiles>COc1ccc(C=O)cc1</smiles>

p-anisaldehyde<smiles>CC(C)=CCC/C(C)=C/CC/C(C)=C/CO</smiles><smiles>C=CCc1ccc(OC)cc1</smiles>

Figure 2: Main aroma compounds of star anise harvested by SDE

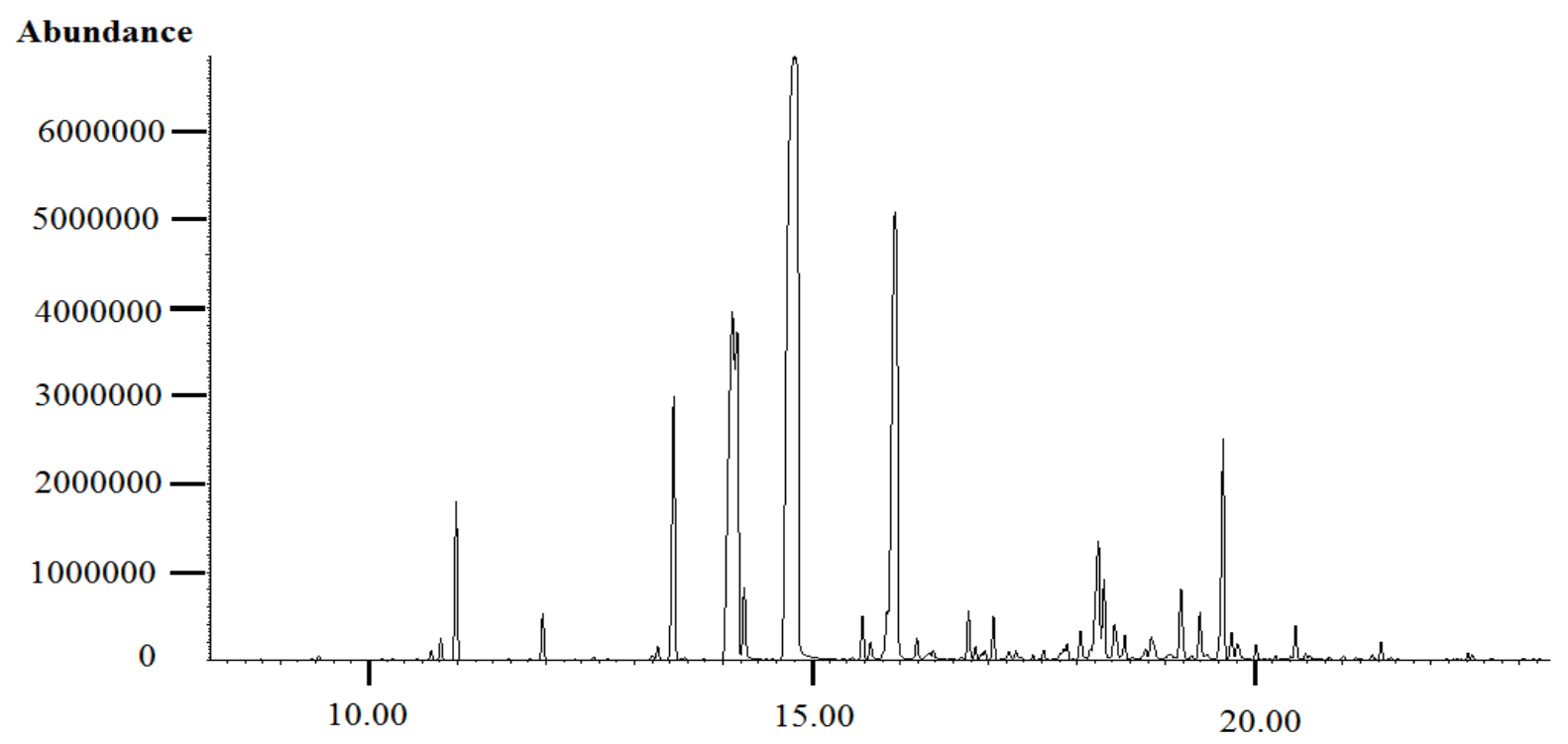

Figure 1: Total ion current chromatogram for star anise SDE extracts. Abscissa: time (min); ordinate: abundance. Data were processed with Agilent GC/MSD Productivity ChemStation 
All the FD values $\left(\log _{3} F D=1\right)$ of $p$ anisaldehyde, farnesol, estragole, limonene and caryophyllene were not more than 1 in total star anise volatile aromatic molecules by GC-O evaluation. Both the FD value $\left(\log _{3} \mathrm{FD}=2\right)$ of trans-anethole and linalool exceeded 2. The FD value of trans-anethole $\left(\log _{3} \mathrm{FD}=5\right)$ was more than 3. Results indicated that the contribution for star anise aroma of trans-anethole was the largest, followed by linalool. In short, these compounds formed characteristic aroma for star anise.

\section{SPME-GC-MS results}

The qualified samples that meet sensory assessment standards were chosen for SPMEGC-MS analysis. For example, among all the main compounds of a batch of star anise sample, the contents (calculated by area normalization method) of linalool, trans-anethole and estragole exceeded $0.5 \%$ (integral surface $>15000000$ ), $55 \%$ (integral surface > 511700000) and $5 \%$ (integral surface $>57000000$ ), respectively.

\section{DISCUSSION}

The composition and proportion of flavor compounds affected the flavor of star anise. However, flavor substances are generally volatile and instable during processing. Thus, collecting volatile molecules is of interest for sensory evaluation and quality control in industry application of star anise. Using liquid $\mathrm{CO}_{2}$, alcohol and/or other solvents to extract volatile substances from star anise were effective [6]. However, these methods usually focus on the recovery or yield of process and the extracts were not always of relevance to food odor profile. Therefore traditional solvent extraction (SE) is not useful for identifying characteristic aroma molecules and determining contributions to global aroma to some extent. Thus, we choose an extraction method yielding a product which is representative of the sample.
Simultaneous distillation-extraction (SDE) and solid-phase microextraction (SPME) are generally applied for GC-O analysis due to adequate reproducibility, representativeness and high efficiency [4]. SDE has the advantage of using a small amount of solvent for extracting a large amount of volatile substances. Although SDE alleviates the instability of the analytes by combining distillation and extraction, heatsensitive compounds or artefacts may appear, given that SDE involves high temperatures [7]. We harvested 78 volatile compounds with SDE, although artefacts could not be totally ruled out due to oxidation and/or pollution [8]. However, artefacts may be negligible in total volatile components, considering volatile molecules of star anise that were well-established in literatures, such as trans-anethole, panisaldehyde, linaloo and limonene account for more than $95 \%$ (Fig. 1). To further validate results from SDE, SPME is also applied to prepare volatile components.

SPME is a method that does not require solvent and relative low temperature, which is useful for extracting molecules with different volatility from those extracted by SDE. Consequently, combining SDE and SPME to isolate odor molecules without analyzing entire cohort of volatile compounds is helpful for enhancing representativeness. In addition, it is speculated that SPME is useful in collecting and monitoring volatile molecule profile changing during spice processing due to its efficiency. Thus, it is promising to use SPME in optimizing spice processing. In short, combining SDE and SPME extracting volatile components is beneficial for mutual validation and offering useful tools for investigating spice processing.

After volatile oil has been collected, mass spectroscopy (MS) and GC-O were used to identify volatile compounds and characteristic aroma molecules respectively. MS, a powerful tool for molecular identification, is applied to the quality control of star anise.

Table 1: Characteristic aroma substances

\begin{tabular}{llll}
\hline $\mathbf{R I}$ & Aroma description & Compound & Log FD $_{3}$ \\
\hline 615 & Lemony & Limonene & $<1$ \\
730 & Orchard-like & Linalool & 3 \\
920 & Aniseed & Estragole & 1 \\
1057 & Aniseed, hawthorn-like & P-anisaldehyde & 1 \\
1170 & Aniseed & Trans-anethole & 5 \\
1250 & Aniseed, acetone, and fruity & 4 '-methoxypropiophenone & $<1$ \\
1690 & Aniseed, flowery and fruity & Farnesol & 1 \\
\hline
\end{tabular}


For example, MS was used for distinguishing Chinese star anise (I. verum) from contaminated or adulterated with Japanese star anise ( $I$. anisatum) or other Illicium species [9].

I. anisatum, a closely related species to I. verum, has ten to thirteen follicles, while the latter usually has eight follicles. Even though the morphological differences between Chinese star anise ( $I$. verum Hook. f.) and poisonous star anise (such as I. lanceolatum A. C. Smith) are distinguishable, it is difficult to discriminate between powdered samples and poisonous samples [2]. Furthermore, MS has no power to identify odor-active compounds from a complicated mixture.

Initially, the extracted compounds were confirmed by GC-MS, a method traditionally applied for the evaluation of volatile molecule contributions to global aroma. After that, GC-O (i.e. GC-sniffing) was selected as a suitable method for identifying main components contributing to the aroma, considering GC-MS fails to confirm which compound is the key one for food aroma. Sniffing is a method determining food aroma intensity, which can sequence aroma compounds according to the aroma strength or their contributions to the global aroma. GC-O has shown to be a valuable method for the selection of odor active compounds from a mixture.

Dilution analysis is a GC-O technique estimating the sensory contribution of single odor active compounds, for instance, AEDA and CharmAnalysis (combined hedonic response measurement). AEDA method, the generally applied GC-O sensing method, was chosen to specify the contribution of odor active substance in star anise [4,10]. AEDA results could be used to understand the star anise aroma and help the quality control. In AEDA, the dilution factor (FD value) is simply the last dilution at which an odor active compound is detected [11].

At the beginning, we applied simultaneous distillation extraction-gas chromatography-mass spectroscopy (SDE-GC-MS) for the structural determination of star anise aroma molecules. Then, we used gas chromatography olfactometry-aroma extract dilution analysis (GCO-AEDA) for determining the flavor dilution factor (FD) values. The FD value of trans-anethole was $81\left(\log _{3} F D \geq 5\right)$ and linalool was more than 9 $\left(\log _{3} \mathrm{FD} \geq 3\right)$ in star anise volatile components analyzed by GC-O, which indicated that transanethole was the most important aroma compound for star anise, followed by linalool.
The FD values of other constituents were less than 1. Next, we applied SPME to analyze standard star anise for correlation its results with SDE-GC-MS and GC-O-AEDA.

To ensure the quality of sample is representative and to minimize factors jeopardizing the stability of volatile components during storage, sensory evaluation should be performed before instrumental analysis. Additionally, sensory evaluation is a convenient, efficient and reliable method for star anise quality control to some extent.

We chose sweet aroma, fruity aroma, alcoholic aroma, woody aroma and caramelic aroma as standards for grading aroma as stated.

These results are useful for establishing sensory evaluation standards for star anise. Quality of star anise affects product quality significantly due to its wide applications. The main quality standard for star anise in China is "National Standard of the People's Republic of China GB/T 7652-2006", which only normalized physical and chemical properties for raw star anise [12]. However, there is no specification for star anise aroma as a spice. Thus, it is difficult to maintain quality of star anise stable during process. Consequently, it is necessary to propose a quality standard for normalizing aroma for this spice as follow.

Star anise volatile essential oil content should exceed $7.5 \%$ considering the practice in factories of China and GB/T 7652-2006 for raw material standards. Star anise standard sample should have characteristic spice aroma, sweet smell and brownish red in its color. Main aroma constituent should be trans-anethole, limonene, estragole, p-anisaldehyde and linalool and their total content should be more than $90 \%$ in star anise essential oil. Notably, the content of transanethole should exceed $75 \%$. Together, star anise that meets both the quality standard of sensory evaluation (eg. AEDA analysis) and the instrumental analysis (such as SPME-GC-MS) was defined as qualified product.

Taken together, methods combining GC-OAEDA and SPME-GC-MS are useful for establishing standards for other spice and food aroma. In addition, analyzing characteristic aroma compounds is beneficial for improving food processing and alleviating some safe concerns in processing and storage. Additionally, SPME-GC-MS may be a convenient way to identify star anise in foods. 


\section{CONCLUSION}

Contribution of trans-anethole $\left(\log _{3} \mathrm{FD}=5\right)$ and linalool $\left(\log _{3} F D=2\right)$ to the global aroma is greater than other volatile molecules, determined by AEDA. Combining SDE-GC-MS and AEDA is helpful for determining volatile components and characteristic aroma constituents. It is useful and robust combining instrumental analysis and sensory evaluation for star anise quality control.

\section{ACKNOWLEDGEMENT}

This project was supported by Guide Project of Science and Technology Research of Henan Education Department, China (no. 12B350006).

\section{REFERENCES}

1. Reineccius G, Peterson D. 3 - Principles of food flavor analysis. Instrumental Assessment of Food Sensory Quality. Abington: Woodhead Publishing; 2013. p. 53-102.

2. Wang GW, Hu WT, Huang BK, Qin LP. Illicium verum: $A$ review on its botany, traditional use, chemistry and pharmacology. J Ethnopharmacol 2011; 136 (1): 1020.

3. Lee AY, Kim HS, Choi G, Moon BC, Chun JM, Kim HK. Optimization of ultrasonic-assisted extraction of active compounds from the fruit of star anise by using response surface methodology. Food Anal Method 2014; 7(8):1661-1670.

4. d'Acampora Zellner B, Dugo P, Dugo G, Mondello L. Gas chromatography-olfactometry in food flavour analysis. J Chromatogr A 2008; 1186(1-2): 123-143.
5. Stone, H.; Bleibaum, R.; Thomas, H.A. Sensory evaluation practices. 4th, Amsterdam: Academic Press; 2012; $p 437$.

6. Tuan $D Q$, Ilangantileket SG. Liquid $\mathrm{CO}_{2}$ extraction of essential oil from Star anise fruits (Illicium verum $H$.). J Food Eng 1997; 31(1): 47-57.

7. Riu-Aumatell M, Vargas L, Vichi S, Guadayol JM, LópezTamames E, Buxaderas S. Characterisation of volatile composition of white salsify (Tragopogon porrifolius L.) by headspace solid-phase microextraction (HS-SPME) and simultaneous distillation-extraction (SDE) coupled to GC-MS. Food Chem 2011; 129(2): 557-564.

8. Chaintreau A. Simultaneous distillation-extraction: from birth to maturity - review. Flavour Fragr J 2001; 16(2): 136-148.

9. Shen $Y$, van Beek TA, Claassen FW, Zuilhof $H$, Chen B, Nielen MWF. Rapid control of Chinese star anise fruits and teas for neurotoxic anisatin by Direct Analysis in Real Time high resolution mass spectrometry. J Chromatogr A 2012; 1259: 179-186.

10. Benzo M, Gilardoni G, Gandini C, Caccialanza G, Vita Finzi $P$, Vidari $G$, Abdo S, Layedra P. Determination of the threshold odor concentration of main odorants in essential oils using gas chromatographyolfactometry incremental dilution technique. J Chromatogr A 2007; 1150(1-2): 131-135.

11. van Ruth SM. Methods for gas chromatographyolfactometry: a review. Biomol Eng 2001; 17/45):121-128.

12. Chen SR, Zhang WP. GB/T 7652-2006. Star anise. Beijing: Standards Press of China, 2006. 